\title{
Recent Developments in Diagnostic Imaging Techniques and Management for Acute Pulmonary Embolism: Multicenter Registry by The Japanese Society of Pulmonary Embolism Research
}

\author{
Masahito SAKUMA, Osamu OKADA*, Mashio NAKAMURA**, Norifumi NAKANISHI***, \\ Yoshiyuki MiYAHARA****, Norikazu YAMADA**, Hirofumi FuJIOKA**, \\ Takayuki KURIYAMA*, Takeyoshi KUnIEDA*****, Tsuneaki SUGIMOTO******, \\ Takeshi NAKANO** and Kunio SHIRATO
}

\begin{abstract}
Objective There are many reports on the diagnosis and management of acute pulmonary embolism (APE), but there have been no investigations concerning the actual conditions in which recent developments in diagnostic imaging techniques and therapies have been applied in clinical practice. The present study was designed to investigate the changes in diagnostic imaging techniques and therapies for APE.

Methods and Patients Three hundred and nine APE patients diagnosed during January 1994-October 1997 (Group 1) were compared with 257 APE patients diagnosed during November 1997-October 2000 (Group 2) in terms of the diagnostic imaging techniques and therapies for APE.

Results Compared with Group 1, pulmonary angiography and contrast-enhanced computed tomography were more frequently performed for diagnosis in Group $2[45.3 \%$ vs $56.8 \%(p=0.0069)$ and $13.9 \%$ vs $57.6 \%$ $(p<0.0001)$, respectively]. Heparin and vena cava filter were used more often in Group $2[74.4 \%$ vs $82.1 \%$ ( $p=0.033)$ and $18.4 \%$ vs $33.9 \%(p<0.0001)$, respectively]. The frequency of thrombolytic therapy was unchanged between the two groups. Warfarin use in discharged patients increased from $71.9 \%$ to $83.8 \% \quad(p=0.0022)$. However, the examination rates for deep vein thrombosis (DVT) were low $(60.8 \%$ in Group 1 and $65.4 \%$ in Group
\end{abstract}

2, $p=0.29$ ) and unchanged using any imaging techniques.

Conclusion The diagnostic imaging techniques for APE increased in variety and the management has improved, while the diagnosis for DVT remains unchanged. (Internal Medicine 42: 470-476, 2003)

Key words: computed tomography, vena cava filter, heparin, thrombolytic therapy, warfarin, deep vein thrombosis

\section{Introduction}

The diagnosis and management for acute pulmonary embolism (APE) have changed in recent years. It is wellrecognized that computed tomography (CT), especially helical CT (1-3) and electron beam tomography $(4,5)$, and magnetic resonance (MR) angiography (6) are useful for the diagnosis of APE. Most of the major hospitals in Japan are equipped with CT and MR. In the management of APE, temporal inferior vena caval filters can be used.

There are many reports on the diagnosis and management of APE $(7,8)$, but there have been no investigations concerning the actual conditions in which recent developments in diagnostic imaging techniques and therapies have been applied in clinical practice.

The Japanese Society of Pulmonary Embolism Research

From Department of Cardiovascular Medicine, Tohoku University Graduate School of Medicine, Sendai, *Department of Chest Medicine, Chiba University, Chiba, **the First Department of Internal Medicine, Mie University, Tsu, ***Department of Internal Medicine, National Cardiovascular Center, Osaka, ****the Second Department of Internal Medicine, Nagasaki University, Nagasaki, *****Department of Medicine, Ise Keio Hospital, Keio University, Ise and $* * * * * *$ Kanto Central Hospital, Tokyo

Received for publication November 26, 2002; Accepted for publication March 12, 2003

Reprint requests should be addressed to Dr. Kunio Shirato, Department of Cardiovascular Medicine, Tohoku University Graduate School of Medicine, 1-1 Seiryo-machi, Aoba-ku, Sendai 980-8574 
(JaSPER) registered cases with pulmonary embolism during two periods (from January 1994 to October 1997 and from November 1997 to October 2000). The present study was designed to investigate the changes in diagnostic imaging techniques and therapies for APE, based on this registry.

\section{Methods}

\section{Study population}

The registry in the present study was conducted first between January 1994 to October 1997 and then between November 1997 to October 2000. In the first survey (9), a total of 533 consecutive Japanese patients with pulmonary embolism were registered from the participating centers in JaSPER (listed in the Appendix), and in the second, there were 373 patients. The diagnosis of pulmonary embolism was made either by a pulmonary angiogram, CT, or MR angiogram which indicated vessel occlusions or intraluminal filling defects, by a lung perfusion scan which indicated the high probability of pulmonary embolism, by transesophageal echocardiogram (TEE), or by an autopsy.

APE was defined as pulmonary embolism which occurs suddenly and we excluded the cases with a mean pulmonary artery pressure of over $40 \mathrm{mmHg}$ without preexisting cardiac or pulmonary disease. There were 309 patients with APE between January 1994 and October 1997 (Group 1) and 257 between November 1997 and October 2000 (Group 2). For statistical analysis, the present study considered only those patients with APE.

All decisions concerning the diagnostic workup and treatment were made by the clinicians caring for each patient. The steering committee took every care not to influence the management strategy employed in the participating hospitals.

\section{Data acquisition}

Complete information on the clinical course and the diag- nostic and therapeutic management of the patients entering the registry was obtained by means of a standardized questionnaire sent to the participating centers by the steering committee. Data were collected on 1) clinical symptoms and signs of the patients at diagnosis; 2) presence of underlying diseases or predisposing factors for APE; 3 ) definitive diagnostic procedures given to patients (pulmonary angiography, perfusion lung scan, contrast-enhanced CT, MR angiography, TEE, and autopsy, and in Group 2, also types of CT); 4) presence of deep venous thrombosis (DVT) and diagnostic procedures (contrast venography, venous ultrasonography, and radionuclide venography); 5) treatment given to patients (anticoagulation, thrombolysis, pulmonary embolectomy, catheter interventional therapy, and inferior vena cava filter implantation, and in Group 2, the types of filters); and 6) in-hospital clinical course.

\section{Statistical analysis}

Statistical analysis was carried out using StatView 5.0 (SAS Institute Inc). All continuous variables were expressed as mean \pm standard deviation and assessed by unpaired t-test. Comparisons of proportion were made by chi-square statistics or, when appropriate, by Fisher's exact test. Multiple logistic regression analysis was used to investigate the independent effect of multiple factors on remarkable facts. The results of the logistic regression models are presented as estimated odds ratios with the corresponding 95\% confidence intervals. All significant tests were two-tailed, with $\mathrm{p}<0.05$ considered as statistically significant.

\section{Results}

The clinical backgrounds of patients with APE had no significant differences between Groups 1 and 2 (Table 1). The mean age of the patients at diagnosis was $59.9 \pm 14.8$ years in Group 1 , and $60.3 \pm 15.5$ in Group $2(\mathrm{p}=0.70)$. The overall in-

Table 1. Selected Characteristics at Diagnosis in Patients with Acute Pulmonary Embolism

\begin{tabular}{lrrr}
\hline \multicolumn{1}{c}{ Characteristics } & Group 1 $(\mathrm{n}=309)$ & Group 2 $(\mathrm{n}=257)$ & p value \\
\hline Age $\geqq 65$ years & $137(44.3 \%)$ & $119(46.3 \%)$ & 0.67 \\
Female & $187(60.5 \%)$ & $162(63.0 \%)$ & 0.54 \\
Cardiogenic shock & $111(35.9 \%)$ & $79(30.7 \%)$ & 0.21 \\
Recent major operation & $110(35.6 \%)$ & $91(35.4 \%)$ & $>0.99$ \\
Recent major trauma or fracture & $29(9.4 \%)$ & $20(7.8 \%)$ & 0.55 \\
Cancer & $70(22.7 \%)$ & $52(20.2 \%)$ & 0.53 \\
Prolonged immobilization & $71(23.0 \%)$ & $55(21.4 \%)$ & 0.69 \\
Obesity (BMI $\geqq 25.3)$ & $106(34.3 \%)$ & $74(28.8 \%)$ & 0.17 \\
Coagulopathy & $17(5.5 \%)$ & $10(3.9 \%)$ & 0.43 \\
Onset in hospital & $145(46.9 \%)$ & $127(49.4 \%)$ & 0.61 \\
\hline
\end{tabular}

Data presented are number $(\%)$ of patients. Recent major operation: any intraabdominal operation and all other operations lasting more than 45 minutes within a month, Recent major trauma or fracture: trauma or fracture involving the pelvis and lower extremities within a month, Prolonged immobilization: immobilization lasting at least 1 week, BMI: body mass index. 


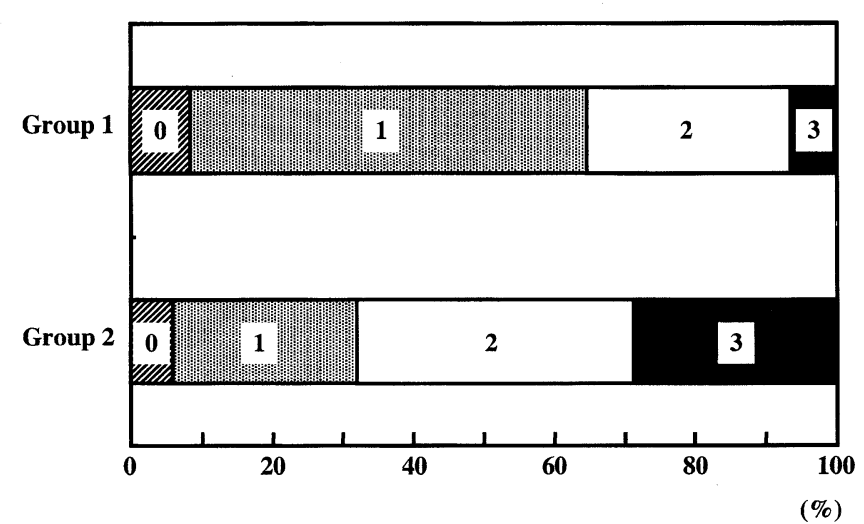

Figure 1. The number of uses of the three main imaging techniques (perfusion lung scan, pulmonary angiography and enhanced computed tomography) employed for diagnosis. 0, 1, 2 and 3 in this figure indicate the number of main diagnostic imaging techniques used. The distribution varied between Groups 1 and $2(p<0.0001)$.

hospital mortality rate was $14.2 \%$ in Group 1 and $11.9 \%$ in Group $2(\mathrm{p}=0.45)$.

\section{Diagnostic imaging techniques for APE}

Perfusion lung scan was performed in $74.1 \%$ of Group 1 and in $76.7 \%$ of Group $2(\mathrm{p}=0.50)$, and ventilation scan in $28.2 \%$ of Group 1 and in $23.0 \%$ of Group 2 ( $p=0.64)$. Compared with Group 1, pulmonary angiography and contrast-enhanced CT were more frequently performed for diagnosis in Group 2 [45.3\% vs 56.8\% (p=0.0069) and $13.9 \%$ vs $57.6 \%(\mathrm{p}<0.0001)$, respectively]. In Group 2, the type of CT in 66 patients $(44.6 \%$ of patients underwent CT) was helical $\mathrm{CT}$, and that in 3 patients $(2.0 \%)$ was electron beam tomography. The use of MR angiography and TEE had increased $(2.3 \%$ in Group 1 vs $6.2 \%$ in Group 2, $\mathrm{p}=0.019$; $0.6 \%$ vs $3.9 \%, \mathrm{p}=0.015$, respectively).

The number of main imaging techniques (perfusion lung scan, pulmonary angiography and CT) used for diagnosis changed between Group 1 and 2 (Fig. 1). In cases that had undergone perfusion lung scan, pulmonary angiography and/or CT were performed in $45.9 \%$ of Group 1 and in
$81.7 \%$ of Group $2(\mathrm{p}<0.0001)$. However, in cases that had not undergone perfusion lung scan, the frequency of pulmonary angiography and/or CT was unchanged between Group 1 and $2(67.5 \%$ vs $75.0 \%, \mathrm{p}=0.36)$.

\section{Therapies for APE}

Heparin was used more often in Group 2 (74.4\% in Group 1 and $82.1 \%$ in Group 2, $p=0.033$ ). The frequency of thrombolytic therapy was unchanged between the two groups (50.2\% in Group 1 vs $48.2 \%$ in Group 2, p=0.67). Catheter interventional therapy, percutaneous cardiopulmonary support, and surgical thrombectomy were unchanged between Group 1 and $2(5.5 \%$ vs $5.8 \%, \mathrm{p}=0.86 ; 1.6 \%$ vs $2.7 \%$, $\mathrm{p}=0.39$; and $2.3 \%$ vs $2.7 \%, \mathrm{p}=0.80$, respectively). Vena cava filters were used more often in Group 2 (18.4\% in Group 1 and $33.9 \%$ in Group 2, p<0.0001). In Group 2, 53 patients (60.9\% of patients used vena cava filters) were implanted with only the permanent type, $28(32.2 \%)$ with the temporary type, and the temporary type was replaced with the permanent type in $6(6.9 \%)$. Warfarin use in discharged patients increased from $71.9 \%$ to $83.8 \%(\mathrm{p}=0.0022)$.

\section{Diagnostic imaging techniques for DVT}

The examination rates for DVT were not so high $(60.8 \%$ in Group 1 and 65.4\% in Group 2, p=0.29) and were unchanged by any of the imaging techniques in cases for which DVT was evaluated [venography $(80.9 \%$ vs $73.2 \%, \mathrm{p}=0.10)$, radionuclide venography $(13.3 \%$ vs $11.9 \%, \mathrm{p}=0.75)$ and venous ultrasonography $(18.1 \%$ vs $23.8 \%, \mathrm{p}=0.19)]$.

\section{Relationship between vena cava filter, and examina-} tion of pulmonary angiography or evaluation of DVT

Vena cava filters were implanted in $37.8 \%$ of the patients that had undergone pulmonary angiography, and in $12.9 \%$ of the patients that did not $(\mathrm{p}<0.0001)$. The examination for DVT was carried out in $73.6 \%$ of patients implanted with vena cava filters, and in $57.8 \%$ of patients not implanted with filters $(p=0.0007)$. Multi-factorial analysis revealed that the practice of pulmonary angiography was related to the implantation of vena cava filters and not to the period of examination, and that the use of vena cava filters was related to both the diagnostic procedures for DVT and the period of examination (Table 2).

Table 2. Results of Multiple-logistic Analysis (n=566)

\begin{tabular}{llrrr}
\hline \multicolumn{1}{c}{ The dependent variable } & The independent variables & Odds ratio & 95\%CI & $\mathrm{p}$ value \\
\hline \multirow{2}{*}{ PAG } & Implantation of vena cava filter & 3.91 & $2.55-5.99$ & $<0.0001$ \\
& Group 2 & 1.33 & $0.94-1.89$ & 0.11 \\
\hline \multirow{2}{*}{ Vena Cava filter } & Examination of DVT & 2.04 & $1.34-3.12$ & 0.001 \\
& Group 2 & 2.25 & $1.53-3.35$ & $<0.0001$ \\
\hline
\end{tabular}

PAG: pulmonary angiography, DVT: deep vein thrombosis, CI: confidence interval. 


\section{Discussion}

Diagnostic imaging techniques for venous thromboembolism have markedly progressed. The recognition of the disease appears to have increased. In the present study, we clarified the clinical state of APE in Japan by examining the progress in diagnostic imaging techniques and management.

\section{Diagnosis of APE}

Diagnostic imaging techniques have come to be used more aggressively. Especially, enhanced CT is used more frequently. Helical CT and electron beam tomography (1-5), which can be performed rapidly and have high resolution, have become popular and their sensitivity and specificity are not inferior to those of pulmonary angiography (1-5). Prospective studies showed that the sensitivity of helical CT ranged from $53 \%$ to $100 \%$ and the specificity from $81 \%$ to $100 \%$ (10). Moreover, CT is less invasive than pulmonary angiography and does not require special techniques. The increase in the use of CT may be due to these advantages.

In both examined periods, perfusion lung scan was performed in about three-fourths of the APE patients, and ventilation scan in about one-fourth. But, as shown in the results from PIOPET study (11), perfusion lung scan has a low specificity and is useful for a definite diagnosis only in limited cases.

Pulmonary angiography use for the diagnosis of APE also increased. This procedure is invasive but can be followed with catheter interventional therapy, including implantation of vena cava filter. Our results indicate the possibility that invasive implantation of vena cava filters may have augmented the use of pulmonary angiography for the diagnosis of APE. In the clinical situation, because management typically follows diagnosis, the idea that the method of management (or prophylaxis) would determine the method of diagnosis is somewhat unusual. But if it can be accepted that the trend toward the use of invasive trans-venous implantation of filter influences the use of pulmonary angiography, the period of occurrence of APE, that is, whether the patients with APE were in Group 1 or in Group 2, would not be related to the frequency of the use of pulmonary angiography (Table 2).

There are many reports about the usefulness of MR angiography for the diagnosis of APE (6). Lobar or segmental pulmonary embolism but not subsegmental embolism can be easily detected by MR angiography (6). In the present study, MR angiography came to be used more frequently, but was employed in only a small number of cases. MR angiography can be used for perfusion imaging (12), but not for patients with medical instruments affected by a strong magnetic field.

TEE is helpful to detect proximal pulmonary embolisms and its usefulness increases in the patients with cardiopulmonary arrest $(13,14)$ because other diagnostic techniques are difficult to use. As shown in the present study, TEE use, although increasing, is still rare.

Several imaging techniques are being used in combination for the diagnosis of APE and this may lead to the increased use of each imaging technique. In the subgroup of patients who underwent perfusion lung scan, the practical rate of contrast-enhanced CT and/or pulmonary angiography is increasing recently, but in the subgroup of patients who did not undergo perfusion lung scan the practical rate of contrastenhanced CT and/or pulmonary angiography did not increase. This suggests that lung perfusion scan is used as a screening procedure for the diagnosis of APE and that, in many recent cases, the diagnosis is confirmed by other imaging techniques after perfusion scan. This may be one of the reasons why contrast-enhanced CT and pulmonary angiography are used more frequently.

\section{Management of APE}

Anticoagulant therapy with intravenous heparin immediately after the onset of APE (15) and oral warfarin sodium are widely accepted for APE patients without contraindications $(16,17)$. Therefore, the recent increase in the use of these medicines appears reasonable. While thrombolytic therapy in clinically unstable cases or cases with overload of the right heart may be acceptable $(18,19)$, there is no clear evidence on the effectiveness of thrombolytic therapy in clinically stable cases without overload of the right heart. One-half of the patients were treated with thrombolytic therapy in both periods. The present study shows that the medical treatment for APE in Japan follows closely the generally accepted recommendations.

Vena cava filters were used more frequently than before. In Group 2, the temporary type increased by one-third in the use of filters. Vena cava filters may prevent re-embolization, which could otherwise lead to critical illness or death early after the onset of APE. Decousus et al showed in their randomized prospective trial that permanent vena cava filters prevented early recurrence of APE but increased chronic DVT (20). One of the reasons for the incremental use of temporary filters in patients with a temporary risk of venous thrombosis is that they protect against the early recurrence of APE without chronic adverse effects on the venous blood flow.

Catheter interventional therapy, percutaneous cardiopulmonary support and surgical embolectomy were used in a small number of the APE patients. But these therapies are important in the cases with cardiopulmonary arrest or in those that are clinically unstable, and may be useful for the patients with contraindications for thrombolysis (21-24). With increasing skill in catheter intervention, advances in the devices, and improving of cooperation with surgeons, these therapies will become widely used for patients who are too severe to benefit from the present system of management.

The selection of both diagnostic imaging techniques to be used and the type of management is greatly affected by the set-up ratio of each type of equipment and the severity of the illness. We could not examine the extent to which each institute that joined the registry possessed the various types of diagnostic equipment. But as shown in Table 1, the patient 
characteristics were not different between Groups 1 and 2.

\section{Mortality for APE}

Mortality for APE was unchanged between the two periods. While the clinical diagnostic rate of APE in Japan was about one 200th that in the United States (25), the annual age-adjusted pulmonary embolism mortality per 100,000 persons in 1996 in Japan based on death certificates $(0.7$ among men and 0.8 among women (26)) was similar to that in non-white and non-black Americans (1.0 among men and 0.7 among women (27)) which is about one-third that in white Americans. In the clinical setting, the prevalence of APE in Asians was about one-fifth that in white Americans, showing the racial difference in the risk for APE (28). But the racial difference cannot completely explain the difference in the clinical diagnostic rate between Japan and America. A low ratio of correct diagnosis of patients with APE in Japan may also be a factor. Moreover, about $30-40 \%$ of the patients in this registry had cardiogenic shock, and this ratio is higher than in Western countries (29). It appears that the less severe cases of APE were under-diagnosed in Japan. If the diagnostic rate is elevated in the future, the mortality will appear to improve even without an actual change.

\section{Imaging techniques of DVT}

From the present results, some problems with the diagnosis of DVT in Japan appeared. First, the examination rates for DVT were low. Second, ultrasonographic techniques, which are non-invasive and have been recognized as useful, were used in only a minority of the patients.

DVT is well-known as one of the main causes of APE (29). But, as shown in previous reports, the detection rate of DVT and the frequency of the signs of DVT are low in Japan $(9,30)$. Therefore, physicians' interest in DVT is low in Japan and the level of research for DVT is also low. However, because one of the reasons for death from APE is acute recurrence, an increase in the detection of DVT may be needed to lower the mortality and, practically, to decide the indication for the use of vena cava filters.

\section{Conclusion}

The diagnostic imaging techniques for APE increased in variety and the management has improved, while the diagnosis for DVT remains unchanged.

\section{Appendix}

The leading members and their clinical centers belonging to JaSPER are listed as follows; Katsuya Akashi: Department of Emergency Medicine, St. Marianna University School of Medicine, Kawasaki; Motomi Ando: Department of Thoracic Surgery, Fujita Health University, Toyoake; Sadahiro Asai: Sasebo City General Hospital, Sasebo; Hirofumi Fujioka: Hakusan Clinic, Hakusan; Satoru Fujita: Takarazuka Daiichi Hospital, Takarazuka; Hisayoshi Fujiwara: The Second Department of Medicine, Gifu University,
Gifu; Nobuo Fukuda: Zentsuji National Hospital, Zentsuji; Hitoshi Furuya: Department of Anesthesiology, Nara Medical School, Kashihara; Koji Goto: Health Care Center, Gifu University, Gifu; Masayuki Hamada: Suzuka Genaral Hospital Suzuka; Shunnosuke Handa: Department of Cardiology, Tokai University Tokyo Hospital, Tokai University, Tokyo; Shinsuke Hiramatsu: Numazu City Hospital, Numa$\mathrm{zu}$; Katsuhiko Hiramori: The Second Department of Internal Medicine, Iwate Medical University, Morioka; Tetsuya Hisada: Tokyo Teishin Hospital, Tokyo; Hitoshi Hishida: Department of Cardiology, Fujita Health University, Toyoake; Norimoto Hoda: Saiseikai Matsusaka General Hospital, Matsusaka; Kan Honda: Anesthesiology and Pain Clinic Division National Cancer Center Hospital, Tokyo; Tadashi Horiuchi: Kanto Central Hospital, Tokyo; Shunichi Hoshino, Fukushima Daiichi Hospital, Fukushima; Takeyuki Hozumi: Department of Internal Medicine and Cardiology, Graduate School of Medicine, Osaka City University, Osaka; Ichiro Inoue: Hiroshima City Hospital, Hiroshima; Kinji Ishikawa: Department of Cardiology, Kinki University, Osakasayama; Minoru Kanazawa: Saitama Cardiovascular and Respiratory Center, Konan; Junji Kanda: Asahi-Chuoh Hospital, Chiba; Michio Kawasuji: The First Department of Surgery, Kumamoto University, Kumamoto; Kenjiro Kikuchi: The First Department of Medicine, Asahikawa Medical College, Asahikawa; Kazuo Kimura: Cardiovascular Center, Yokohama City University Medical Center, Yokohama; Takao Kobayashi: Department of Obstetrics and Gynecology, Hamamatsu University School of Medicine, Hamamatsu; Tokuji Konishi: Mie Prefectural General Medical Center, Yokkaichi; Shigeru Kohno: The Second Department of Internal Medicine, Nagasaki University, Nagasaki; Isao Kubota: The First Department of Internal Medicine, Yamagata University, Yamagata; Takeyoshi Kunieda: Sumida Shuko-en Facility, Tokyo; Sachio Kuribayashi: Department of Radiology, Keio University, Tokyo; Takayuki Kuriyama: Department of Respirology, Graduate School of Medicine, Chiba University, Chiba; Masayuki Kuroiwa: Department of Anesthesiology, Kitazato University, Sagamihara; Masahisa Masuda: National Chiba Hospital, Chiba; Rokuro Matsuoka: Showa General Hospital, Tokyo; Yoshihiko Miake: Department of Cardiology, St. Marianna University School of Medicine, Kawasaki; Syogo Misawa: Tokyo Medical Examiner's Office, Tokyo; Mitsuhiro Mochizuki: Komaki Citizen Hospital, Komaki; Shinichi Momomura: Toranomon Hospital, Tokyo; Hajime Morikawa: Department of Obstetrics and Gynecology, Nara Medical School, Kashihara; Hiroshi Morio: Narita Red Cross Hospital, Narita; Shigefumi Morioka: Kobe City General Hospital, Kobe; Takeshi Morishita: Ohfuna Central Hospital, Kamakura; Mitsuru Munakata, Department of Pulmonary Medicine, Fukushima Medical University, Fukushima; Takanori Murayama: Department of Anesthesiology, Jichi Medical School Omiya Medical Center, Saitama; Hideo Nagai: Department of Surgery, Jichi Medical School, Minamikawachi; Ryozo Nagai: Department of Cardiovascular Medicine, Graduate 


\section{Clinical Progress in Pulmonary Embolism}

School of Medicine, University of Tokyo, Tokyo; Yasushi Nakajima: Department of Radiology, St. Marianna University School of Medicine, Kawasaki; Takaaki Nakamoto: Department of Cardiology and Pneumology, Dokkyo University School of Medicine, Mibu; Norifumi Nakanishi: Department of Internal Medicine, National Cardiovascular Center, Osaka; Takeshi Nakano: The First Department of Internal Medicine, Mie University, Tsu; Masaharu Nishimura: Department of Respiratory Medicine, Graduate School of Medicine, Hokkaido University, Sapporo; Akihiro Niwa: Musashino Red Cross Hospital, Musashino; Hiroshi Niwa: Seirei Mikatahara General Hospital, Hamamatsu; Satoshi Ogawa: Department of Medicine, Keio University, Tokyo; Hitoshi Ogino: Department of Cardiovascular Surgery, National Cardiovascular Center, Osaka; Shigetsugu Ohgi: The Second Department of Surgery, Tottori University, Yonago; Yoshiaki Okano: Department of Clinical Laboratory Medicine, Graduate School of Medicine, Kyoto University, Kyoto; Ken Okumura: The Second Department of Internal Medicine, Hirosaki University, Hirosaki; Muneyasu Saito: Department of Cardiology, Jichi Medical School Omiya Medical Center, Saitama; Masato Sakon: Department of Surgery and Clinical Oncology, Graduate School of Medicine, Osaka University, Osaka; Harumi Sakurada: Metropolitan Hiroo General Hospital, Tokyo; Toshitami Sawayama: Sawayama Clinic, Kurashiki; Noritada Seo: Department of Anesthesiology and Critical Care Medicine, Jichi Medical School, Minamikawachi; Takahiro Shibata: Department of Cardiology, Jikei University School of Medicine, Tokyo; Tetsuo Shibata: Ichinomiya City Hospital, Ichinomiya; Kiyokazu Shimizu: Handa City Hospital, Handa; Kunio Shirato: Department of Cardiovascular Medicine, Graduate School of Medicine, Tohoku University, Sendai; Takahito Sone: Ogaki Municipal Hospital, Ogaki; Tsuneaki Sugimoto: Kanto Central Hospital, Tokyo; Hajime Suzuki: Department of Respiratory Internal Medicine, Showa University Fujisawa Hospital, Showa University, Yokohama; Hiroyuki Tajima: Department of Radiology Medicine, Nippon Medical School, Tokyo; Keigo Takagi: Respiratory Center, Toho University Omori Hospital, Toho University Tokyo; Shinichi Takamoto: Department of Cardiovascular Surgery, Graduate School of Medicine, University of Tokyo, Tokyo; Morimasa Takayama: The First Department of Internal Medicine, Nippon Medical School, Tokyo; Kan Takeda: Department of Radiology, Mie University, Tsu; Teruhisa Tanabe: Department of Cardiology, Tokai University, Isehara; Keiji Tanaka: Intensive and Coronary Care Unit, Nippon Medical School, Tokyo; Shinichi Toyama: Kanagawa Prefectural Cardiovascular and Respiratory Center, Yokohama; Atsumasa Uchida: Department of Orthopedic Surgery, Mie University, Tsu; Hideo Wada: Department of Laboratory Medicine, Mie University, Tsu; Sachiro Watanabe: Gifu Prefectural Gifu Hospital, Gifu; Hideya Yamamoto: The Second Department of Internal Medicine, Hiroshima University, Hiroshima; Junichi Yamazaki: Department of Cardiology, Toho University
Omori Hospital, Toho University, Tokyo; Osamu Yanase: Tokyo Metropolitan Otsuka Hospital, Tokyo; Mitsuhiro Yokoyama: Division of Cardiovascular and Respiratory Medicine, Graduate School of Medicine, Kobe University, Kobe; Kiyoshi Yoshida: Department of Cardiology, Kawasaki Medical School, Kurashiki; Chikao Yutani: Department of Pathology, National Cardiovascular Center, Osaka.

\section{References}

1) Lorut C, Ghossains M, Horellou MH, Achkar A, Fretault J, Laaban JP. A noninvasive diagnostic strategy including spiral computed tomography in patients with suspected pulmonary embolism. Am J Respir Crit Care Med 162: 1413-1418, 2000.

2) Pruszczyk P, Torbicki A, Pacho R, et al. Noninvasive diagnosis of suspected severe pulmonary embolism: transesophageal echocardiography vs spiral CT. Chest 112: 722-728, 1997.

3) Remy-Jardin M, Remy J, Deschildre F, et al. Diagnosis of pulmonary embolism with spiral CT: comparison with pulmonary angiography and scintigraphy. Radiology 200: 699-706, 1996.

4) Teigen CL, Maus TP, Sheedy PF II, Johnson CM, Stanson AW, Welch TJ. Pulmonary embolism: diagnosis with electron-beam CT. Radiology 188: 839-845, 1993.

5) Teigen CL, Maus TP, Sheedy PF II, et al. Pulmonary embolism: diagnosis with contrast-enhanced electron-beam CT and comparison with pulmonary angiography. Radiology 194: 313-319, 1995.

6) Gupta A, Frazer CK, Ferguson JM, et al. Acute pulmonary embolism: diagnosis with MR angiography. Radiology 210: 353-359, 1999.

7) Tai NR, Atwal AS, Hamilton G. Modern management of pulmonary embolism. Br J Surg 86: 853-868, 1999.

8) Arcasoy SM, Kreit JW. Thrombolytic therapy of pulmonary embolism: a comprehensive review of current evidence. Chest 115: 1695-1707, 1999.

9) Nakamura M, Fujioka H, Yamada N, et al. Clinical characteristics of acute pulmonary thromboembolism in Japan: results of a multicenter registry in the Japanese Society of Pulmonary Embolism Research. Clin Cardiol 24: 132-138, 2001.

10) Rathbun SW, Raskob GE, Whitsett TL. Sensitivity and specificity of helical computed tomography in the diagnosis of pulmonary embolism: a systematic review. Ann Intern Med 132: 227-232, 2000.

11) The PIOPED Investigators. Value of the ventilation/perfusion scan in acute pulmonary embolism; results of the prospective investigation of pulmonary embolism diagnosis (PIOPED). JAMA 263: 2753-2759, 1990.

12) Hatabu H, Gaa J, Kim D, Li W, Prasad PV, Edelman RR. Pulmonary perfusion: qualitative assessment with dynamic contrast-enhanced MRI using ultra-short TE and inversion recovery turbo FLASH. Magn Reson Med 36: 503-508, 1996.

13) Vieillard-Baron A, Qanadli SD, Antakly Y, et al. Transesophageal echocardiography for the diagnosis of pulmonary embolism with acute cor pulmonale: a comparison with radiological procedures. Intensive Care Med 24: 429-433, 1998.

14) Krivec B, Voga G, Zuran I, et al. Diagnosis and treatment of shock due to massive pulmonary embolism: approach with transesophageal echocardiography and intrapulmonary thrombolysis. Chest 112: 13101316, 1997.

15) Barritt DW, Jordan SC. Anticoagulant drugs in the treatment of pulmonary embolism: a controlled trial. Lancet i: 1309-1312, 1960.

16) Lagerstedt CI, Olsson CG, Fagher BO, Oqvist BW, Albrechtsson U. Need for long-term anticoagulant treatment in symptomatic calf deepvein thrombosis. Lancet ii: 515-518, 1985.

17) Research Committee of the British Thoracic Society. Optimum duration of anticoagulation for deep-vein thrombosis and pulmonary embolism. Lancet 340: 873-876, 1992.

18) Jerjez-Sanchez C, Ramirez-Rivera A, de Lourdes Garcia $M$, et al. 


\section{SAKUMA et al}

Streptokinase and heparin versus heparin alone in massive pulmonary embolism: a randomized controlled trial. J Thromb Thrombolys 2: 227-229, 1995.

19) Konstantinides S, Geibel A, Olschewski M, et al. Association between thrombolytic treatment and the prognosis of hemodynamically stable patients with major pulmonary embolism: results of a multicenter registry. Circulation 96: 882-888, 1997.

20) Decousus H, Leizorovicz A, Parent F, et al. A clinical trial of vena caval filters in the prevention of pulmonary embolism in patients with proximal deep-vein thrombosis. N Engl J Med 338: 409-415, 1998.

21) Stulz P, Schlapfer R, Feer R, Habicht J, Gradel E. Decision making in the surgical treatment of massive pulmonary embolism. Eur J Cardiothorac Surg 8: 188-193, 1994.

22) Ohteki H, Norita H, Sakai M, Narita Y. Emergency pulmonary embolectomy with percutaneous cardiopulmonary bypass. Ann Thorac Surg 63: 1584-1586, 1997.

23) Schmitz-Rode T, Janssens U, Schild HH, Basche S, Hanrath P, Gunther RW. Fragmentation of massive pulmonary embolism using a pigtail rotation catheter. Chest 114: 1427-1436, 1998.
24) Greenfield LJ, Proctor MC, Williams DM, Wakefield TW. Long-term experience with transvenous catheter pulmonary embolectomy. J Vasc Surg 18: 450-457, 1993.

25) Kumasaka N, Sakuma M, Shirato K. Incidence of pulmonary thromboembolism in Japan. Jpn Circ J 63: 439-441, 1999.

26) Sakuma M, Konno Y, Shirato K. Increasing mortality from pulmonary embolism in Japan, 1951-2000. Circ J 66: 1144-1149, 2002.

27) Lilienfeld DE. Decreasing mortality from pulmonary embolism in the United States. Int J Epidemiol 29: 465-469, 2000.

28) Klatsky AL, Armstrong MA, Poggi J. Risk of pulmonary embolism and/or deep venous thrombosis in Asian-Americans. Am J Cardiol 85: 1334-1337, 2000.

29) Stein PD, Willis PW III, DeMets DL. History and physical examination in acute pulmonary embolism in patients without preexisting cardiac or pulmonary disease. Am J Cardiol 47: 218-223, 1981.

30) Kumasaka N, Sakuma M, Shirato K. Clinical features and predictors of in-hospital mortality in patients with acute and chronic pulmonary thromboembolism. Intern Med 39: 1038-1043, 2000. 